\title{
Situación epidemiológica actual del VIH/SIDA en Latinoamérica en la primera década del siglo XXI. Análisis de las diferencias entre países
}

\author{
INMACULADA TEVA ${ }^{\mathrm{a}}, \mathrm{M}^{\mathrm{a}}$ PAZ BERMÚDEZ ${ }^{\mathrm{a}}$, \\ $M^{\text {a TERESA RAMIRO }}{ }^{\mathrm{a}}$, GUALBERTO BUELA-CASAL ${ }^{\mathrm{a}}$
}

Facultad de Psicología. Universidad de Granada (España). apsicologo

No se declaran conflictos de intereses.

Recibido el 28 de julio de 2011, aceptado el 25 de octubre de 2011.

Correspondencia a: Inmaculada Teva Dpto. Psicología Evolutiva y de la Educación Facultad de Psicología Universidad de Granada 18011 Granada (España) Teléfono: +34 958249037 E-mail: inmate@ugr.es

\section{Current epidemiological situation of HIV/AIDS in Latin America. Analysis of differences among countries}

Background: There is a great regional heterogeneity in the prevalence of HIVI AIDS. Aim: To analyze the current situation of HIV/AIDS in Latin America by means of a comparative analysis among countries. Material and Methods: In a descriptive study (document analysis), epidemiological data about HIV/AIDS in Latin America as well as population data were used. Prevalence indexes and the distribution of HIV/AIDS cases were compared according to gender, age and transmission mode. Results: Dominican Republic, Panama, El Salvador and Honduras are the countries with the highest prevalence indexes. Colombia is in the first position in percentage of young men with HIV/AIDS and it is in the second position in percentage of children. Costa Rica is the second country for percentage of young men and male adults infected with HIV/AIDS. Paraguay is in the second place for children with HIV/AIDS and it is the third country in young men living with HIV/AIDS. Dominican Republic is in the first position in the percentage of young and adult females living with HIV/AIDS. The main transmission mode in all Latin American countries is sexual intercourse (heterosexual and homo/bisexual). Conclusions: Latin America is a heterogeneous region in HIV/AIDS which should be considered in the development and establishment of prevention strategies.

(Rev Med Chile 2012; 140: 50-58).

Key words: Acquired immunodeficiency syndrome; Latin America.
L a epidemia por el VIH/SIDA presenta una heterogeneidad a nivel regional, produciéndose un descenso en la prevalencia de VIH/ SIDA en unas zonas (por ejemplo, África subsahariana) y un aumento de la misma en otras (por ejemplo, Europa oriental y Asia central $)^{1}$. Además, continúa en aumento la cantidad de personas que viven con el VIH/SIDA ${ }^{2}$ debido, en parte, a los tratamientos antirretrovirales. Según las últimas estimaciones realizadas, en el mundo hay 33,3 millones de personas que padecen el VIH/SIDA y se produjeron 2,6 millones de nuevas infecciones durante el año 2009.

Un resumen de la situación epidemiológica actual del VIH/SIDA en el mundo en adultos (personas de entre 15 y 49 años, según ONUSIDA) puede observarse en la Tabla 1. Como se muestra, en cuarto lugar se encuentra Latinoamérica mientras que Europa occidental y Norteamérica ocupan las últimas posiciones. Se deduce, por tanto, que las regiones más desfavorecidas, con un menor nivel de ingresos económicos y de desarrollo son las 
Situación epidemiológica actual del VIH/SIDA en Latinoamérica - I. Teva et al

que se encuentran más afectadas por la epidemia. Con respecto a la principal vía de transmisión, en la mayoría de las regiones predomina la vía homosexual (varones que tienen relaciones sexuales con varones) (Tabla 1). Latinoamérica, Norteamérica y Europa occidental comparten esta principal vía de transmisión, lo cual puede deberse a las influencias en el estilo de vida entre estas áreas geográficas ${ }^{3}$. Es más, según la información proporcionada por ONUSIDA $^{2}$ se está registrando en la actualidad un aumento de los casos de VIH en homosexuales en estas zonas, evidenciando un incremento de las conductas de riesgo en esta población. Igualmente, el miedo al estigma y al rechazo en los homosexuales les puede llevar a mantener relaciones sexuales con mujeres, lo cual fomentaría la transmisión del VIH por vía heterosexual ${ }^{2}$ formándose un puente de transmisión del virus entre estos grupos. Se estima que en Latinoamérica la prevalencia de relaciones heterosexuales en homosexuales se encuentra en torno al $30 \%{ }^{4}$.

El interés por la situación epidemiológica del VIH/SIDA se hace patente en las numerosas investigaciones que se han realizado al respecto en los últimos años tanto a nivel global como regional ${ }^{1,3,5-13}$.

El objetivo del presente trabajo es analizar la situación actual del VIH/SIDA en Latinoamérica de manera que se actualiza el artículo de BuelaCasal et $\mathrm{al}^{3}$. Esto es importante dado que tras diez años de la publicación de ese estudio se prevé una evolución en los datos epidemiológicos que son de utilidad a los profesionales que se dedican a la investigación sobre el VIH/SIDA.

Tabla 1. Distribución mundial del VIH/SIDA, clasificando las regiones en función de la tasa de prevalencia. Datos actualizados hasta diciembre de 2010. Fuente: ONUSIDA²

\begin{tabular}{|c|c|c|c|c|c|}
\hline Región & $\begin{array}{c}\text { Tasa de } \\
\text { prevalencia } \\
\text { en adultos } \\
\text { (15-49 años) } \\
(\%)\end{array}$ & $\begin{array}{c}n \text { total } \\
\text { adultos } \\
\text { (15-49 años) }\end{array}$ & $\begin{array}{c}\text { n mujeres } \\
\text { (adultas) } \\
(\%)\end{array}$ & $\begin{array}{c}\text { n varones } \\
\text { (adultos) } \\
(\%)\end{array}$ & $\begin{array}{l}\text { Principal vía de } \\
\text { transmisión }\end{array}$ \\
\hline África Subsahariana & 5,0 & 20.300 .000 & $\begin{array}{r}12.100 .000 \\
(60,0)\end{array}$ & $\begin{array}{r}8.200 .000 \\
(40,0)\end{array}$ & Heterosexual \\
\hline Caribe & 1,0 & 220.000 & $\begin{array}{r}120.000 \\
(54,5)\end{array}$ & $\begin{array}{r}100.000 \\
(45,5)\end{array}$ & Heterosexual \\
\hline Este europeo y Asia central & 0,8 & 1.400 .000 & $\begin{array}{r}690.000 \\
(49,3)\end{array}$ & $\begin{array}{r}710.000 \\
(50,7)\end{array}$ & UDVP \\
\hline Latinoamérica & 0,5 & 1.400 .000 & $\begin{array}{r}490.000 \\
(35,0)\end{array}$ & $\begin{array}{r}910.000 \\
(65,0)\end{array}$ & Homosexual \\
\hline Asia Este & 0,3 & 760.000 & $\begin{array}{r}220.00 \\
(29,0)\end{array}$ & $\begin{array}{r}540.000 \\
(71,0)\end{array}$ & Homosexual \\
\hline Asia Sur y Sureste & 0,3 & 4.000 .000 & $\begin{array}{r}1.400 .000 \\
(35,0)\end{array}$ & $\begin{array}{r}2.600 .000 \\
(65,0)\end{array}$ & Homosexual \\
\hline Oceanía & 0,3 & 54.000 & $\begin{array}{r}25.000 \\
(46,3)\end{array}$ & $\begin{array}{r}29.000 \\
(53,7)\end{array}$ & Heterosexual \\
\hline África Norte y Oriente medio & 0,2 & 440.000 & $\begin{array}{r}210.000 \\
(47,7)\end{array}$ & $\begin{array}{r}230.000 \\
(52,3)\end{array}$ & UDVP \\
\hline Europa occidental y central & 0,2 & 820.000 & $\begin{array}{r}240.000 \\
(29,3)\end{array}$ & $\begin{array}{r}580.000 \\
(70,7)\end{array}$ & Homosexual \\
\hline Norteamérica & 0,2 & 1.500 .000 & $\begin{array}{r}390.000 \\
(26,0)\end{array}$ & $\begin{array}{r}1.110 .000 \\
(74,0)\end{array}$ & Homosexual \\
\hline
\end{tabular}

Nota. Aquellas regiones con idénticas tasas de prevalencia han sido clasificadas por orden alfabético. UDVP: Usuarios de drogas por vía parenteral. 


\section{Material y Método}

Las unidades de análisis fueron los datos acumulados sobre VIH/SIDA proporcionados por ONUSIDA (actualizados a diciembre de 2010) y el Centro Nacional de Epidemiología español (actualizados a 30 de junio de 2010) ${ }^{14}$ así como los datos de población obtenidos a través del Centro Latinoamericano y Caribeño de Demografía (CELADE).

En cuanto al diseño, se trata de un estudio descriptivo mediante observación (análisis de documentos) según la clasificación propuesta por Montero y León ${ }^{15}$.

En relación al procedimiento seguido, en primer lugar se realizó una búsqueda de los datos epidemiológicos más actualizados sobre VIH/SIDA en España y Latinoamérica en las páginas web de ONUSIDA y del Centro Nacional de Epidemiología español. Los índices de prevalencia fueron calculados teniendo en cuenta los casos de VIH/SIDA, obtenidos de ONUSIDA ${ }^{16}$ y los datos poblacionales de cada país, proporcionados por CELADE ${ }^{17}$. La información relativa a las vías de transmisión, ha sido construida utilizando la última información disponible en ONUSIDA, actualizada a marzo de $2010^{18}$. Los datos relacionados con los casos de VIH/SIDA en los países latinoamericanos en función del sexo y la edad fueron elaborados a partir de la información proporcionada por ONUSIDA $^{2}$. A partir de los porcentajes de prevalencia de varones y mujeres jóvenes (personas de entre 15 y 24 años, según ONUSIDA) de los que informa ONUSIDA ${ }^{2}$ y los datos de población de estos grupos de edad en cada país proporcionados por CELADE ${ }^{17}$, se calculó el número de mujeres y varones jóvenes con VIH/SIDA en cada país latinoamericano. Para el cálculo del número de casos de VIH/SIDA en la infancia (personas de entre $0 \mathrm{y}$ 14 años, según ONUSIDA) en cada país, se restó al número total de adultos (personas de entre 15 y 49 años, de acuerdo a ONUSIDA) y niños con VIH/SIDA, el número de adultos con VIH/SIDA. A partir de este dato, se calculó el porcentaje de niños con VIH/SIDA teniendo en cuenta la población infantil de cada país.

\section{Resultados}

La prevalencia y el número total de casos de VIH/SIDA en los países latinoamericanos y España se puede observar en la Tabla 2. Considerando los índices de prevalencia, se han establecido tres grupos de países: países con prevalencia baja (entre 0,5 y 2,4/1.000 habitantes): Chile (2,3), Costa Rica $(2,1)$, México $(2,0)$, Paraguay $(2,0)$, Bolivia $(1,2)$, Nicaragua $(1,2)$ y Cuba $(0,6)$; países con prevalencia media (entre 2,5 y 4,4/1.000 habitantes): Guatemala $(4,3)$, Brasil $(4,1)$, Colombia $(3,5)$, Uruguay $(2,9)$, Argentina $(2,7)$, Ecuador $(2,7)$ y Perú $(2,5)$; países con prevalencia alta (entre 4,5 y 6,4/1.000 habitantes): República Dominicana $(5,8)$, Panamá $(5,7)$, El Salvador $(5,5)$ y Honduras $(5,1)$.

\section{Tabla 2. Prevalencia y número total de casos de VIH/SIDA en los países latinoamericanos y España (actualizado a diciembre de 2010). Fuente: ONUSIDA ${ }^{16}$ y CELADE ${ }^{17}$}

\begin{tabular}{|lrc|}
\hline *Países & $\begin{array}{c}\text { Casos de } \\
\text { VIH/SIDA } \\
\text { (n) }\end{array}$ & $\begin{array}{c}\text { Prevalencia } \\
\text { por cada } \\
\mathbf{1 . 0 0 0} \\
\text { habitantes }\end{array}$ \\
\hline República Dominicana & 57.000 & 5,8 \\
\hline Panamá & 20.000 & 5,7 \\
\hline El Salvador & 34.000 & 5,5 \\
\hline Honduras & 39.000 & 5,1 \\
\hline Guatemala & 62.000 & 4,3 \\
\hline Brasil & 810.000 & 4,1 \\
\hline Colombia & 160.000 & 3,5 \\
\hline Uruguay & 9.900 & 2,9 \\
\hline Argentina & 110.000 & 2,7 \\
\hline Ecuador & 37.000 & 2,7 \\
\hline Perú & 75.000 & 2,5 \\
\hline Chile & 40.000 & 2,3 \\
\hline Costa Rica & 9.800 & 2,1 \\
\hline México & 220.000 & 2,0 \\
\hline Paraguay & 13.000 & 2,0 \\
\hline Bolivia & 12.000 & 1,2 \\
\hline Nicaragua & 6.900 & 1,2 \\
\hline Cuba & 7.100 & 0,6 \\
\hline Venezuela & & - \\
\hline España & 130.000 & 2,9 \\
\hline Nota. Las & $(-1,5$ \\
\hline
\end{tabular}

*Nota. Las casillas con guión (-) se refieren a que no hay datos. Aquellos países con idénticos índices de prevalencia han sido clasificados por orden alfabético. 
En la Tabla 3, se presenta los casos de VIH/ SIDA en los países latinoamericanos en función del sexo y la edad. México (73,2\%), Costa Rica $(69,4 \%)$ y Panamá $(68,5 \%)$ son los países de Latinoamérica con mayor porcentaje de varones adultos con VIH/SIDA. República Dominicana
(56,1\%), Argentina (32,7\%) y El Salvador $(32,4 \%)$ son los países con mayor porcentaje de mujeres adultas que viven con el VIH/SIDA en Latinoamérica. Por otra parte, República Dominicana $(69,8 \%)$, Nicaragua $(50,0 \%)$ y Ecuador $(49,3 \%)$ son los tres países con mayor porcentaje de mujeres

Tabla 3. Casos de VIH/SIDA en los países latinoamericanos por sexo y edad. Fuente: ONUSIDA $^{2}$

\begin{tabular}{|c|c|c|c|c|c|c|c|c|}
\hline País & $\begin{array}{c}\text { n Total } \\
\text { adultos } \\
\text { y niños }\end{array}$ & $\begin{array}{c}n \\
\text { adultos } \\
(15 \text { y } \\
\text { más } \\
\text { años) }\end{array}$ & $\begin{array}{c}\text { Niños } \\
(0-14 \\
\text { años) } \\
\text { n (\%) }\end{array}$ & $\begin{array}{c}\text { Varones } \\
\text { adultos } \\
\text { (15 y más } \\
\text { años) } \\
\text { n (\%) }\end{array}$ & $\begin{array}{l}\text { Mujeres } \\
\text { adultas } \\
\text { (15 y más } \\
\text { años) } \\
\text { n (\%) }\end{array}$ & $\begin{array}{c}\text { n Total } \\
\text { adultos } \\
\text { jóvenes } \\
\text { (15-24 } \\
\text { años) }\end{array}$ & $\begin{array}{c}\text { Mujeres } \\
\text { jóvenes } \\
\text { (15-24 } \\
\text { años) } \\
\text { n(\%) }\end{array}$ & $\begin{array}{c}\text { Varones } \\
\text { jóvenes } \\
\text { (15-24 } \\
\text { años) } \\
\text { n (\%) }\end{array}$ \\
\hline Argentina & 110.000 & 110.000 & - & $\begin{array}{r}74.000 \\
(67,3)\end{array}$ & $\begin{array}{r}36.000 \\
(32,7)\end{array}$ & 17.120 & $\begin{array}{l}6.730 \\
(39,3)\end{array}$ & $\begin{array}{r}10.390 \\
(60,7)\end{array}$ \\
\hline Bolivia & 12.000 & 11.000 & $\begin{array}{r}1.000 \\
(8,3)\end{array}$ & $\begin{array}{l}7.400 \\
(61,7)\end{array}$ & $\begin{array}{l}3.600 \\
(30,0)\end{array}$ & 2.003 & $\begin{array}{r}986 \\
(49,2)\end{array}$ & $\begin{array}{l}1.017 \\
(50,8)\end{array}$ \\
\hline Brasil & - & - & - & - & - & - & - & - \\
\hline Chile & 40.000 & 39.000 & $\begin{array}{r}1.000 \\
(2,5)\end{array}$ & $\begin{array}{r}27.000 \\
(67,5)\end{array}$ & $\begin{array}{r}12.000 \\
(30,0)\end{array}$ & 4.449 & $\begin{array}{l}1.452 \\
(32,6)\end{array}$ & $\begin{array}{l}2.997 \\
(67,4)\end{array}$ \\
\hline Colombia & 160.000 & 150.000 & $\begin{array}{r}10.000 \\
(6,3)\end{array}$ & $\begin{array}{r}100.000 \\
(62,5)\end{array}$ & $\begin{array}{r}50.000 \\
(31,2)\end{array}$ & 12.587 & $\begin{array}{l}4.006 \\
(31,8)\end{array}$ & $\begin{array}{l}8.581 \\
(68,2)\end{array}$ \\
\hline Costa Rica & 9.800 & 9.600 & $\begin{array}{r}200 \\
(2,0)\end{array}$ & $\begin{array}{l}6.800 \\
(69,4)\end{array}$ & $\begin{array}{l}2.800 \\
(28,6)\end{array}$ & 1.336 & $\begin{array}{r}428 \\
(32,0)\end{array}$ & $\begin{array}{r}908 \\
(68,0)\end{array}$ \\
\hline Cuba & 7.100 & 7.000 & $\begin{array}{r}100 \\
(1,4)\end{array}$ & $\begin{array}{l}4.800 \\
(67,6)\end{array}$ & $\begin{array}{l}2.200 \\
(31,0)\end{array}$ & 1.599 & $\begin{array}{r}776 \\
(48,5)\end{array}$ & $\begin{array}{r}823 \\
(51,5)\end{array}$ \\
\hline Ecuador & 37.000 & 36.000 & $\begin{array}{r}1.000 \\
(2,7)\end{array}$ & $\begin{array}{r}25.000 \\
(67,6)\end{array}$ & $\begin{array}{r}11.000 \\
(29,7)\end{array}$ & 5.141 & $\begin{array}{l}2.534 \\
(49,3)\end{array}$ & $\begin{array}{l}2.607 \\
(50,7)\end{array}$ \\
\hline El Salvador & 34.000 & 32.000 & $\begin{array}{r}2.000 \\
(5,8)\end{array}$ & $\begin{array}{r}21.000 \\
(61,8)\end{array}$ & $\begin{array}{r}11.000 \\
(32,4)\end{array}$ & 4.407 & $\begin{array}{l}1.941 \\
(44,0)\end{array}$ & $\begin{array}{l}2.466 \\
(56,0)\end{array}$ \\
\hline Guatemala & 62.000 & 60.000 & $\begin{array}{r}2.000 \\
(3,2)\end{array}$ & $\begin{array}{r}40.000 \\
(64,5)\end{array}$ & $\begin{array}{r}20.000 \\
(32,3)\end{array}$ & 11.619 & $\begin{array}{l}4.415 \\
(38,0)\end{array}$ & $\begin{array}{l}7.204 \\
(62,0)\end{array}$ \\
\hline Honduras & 39.000 & 37.000 & $\begin{array}{r}2.000 \\
(5,1)\end{array}$ & $\begin{array}{r}25.000 \\
(64,1)\end{array}$ & $\begin{array}{r}12.000 \\
(30,8)\end{array}$ & 4.063 & $\begin{array}{l}1.609 \\
(39,6)\end{array}$ & $\begin{array}{l}2.454 \\
(60,4)\end{array}$ \\
\hline México & 220.000 & 220.000 & - & $\begin{array}{r}161.000 \\
(73,2)\end{array}$ & $\begin{array}{r}59.000 \\
(26,8)\end{array}$ & 29.926 & $\begin{array}{r}10.135 \\
(33,9)\end{array}$ & $\begin{array}{r}19.791 \\
(66,1)\end{array}$ \\
\hline Nicaragua & 6.900 & 6.700 & $\begin{array}{r}200 \\
(2,9)\end{array}$ & $\begin{array}{l}4.600 \\
(66,7)\end{array}$ & $\begin{array}{l}2.100 \\
(30,4)\end{array}$ & 1.247 & $\begin{array}{r}624 \\
(50,0)\end{array}$ & $\begin{array}{r}623 \\
(50,0)\end{array}$ \\
\hline Panamá & 20.000 & 20.000 & - & $\begin{array}{r}13.700 \\
(68,5)\end{array}$ & $\begin{array}{l}6.300 \\
(31,5)\end{array}$ & 2.133 & $\begin{array}{r}895 \\
(42,0)\end{array}$ & $\begin{array}{l}1.238 \\
(58,0)\end{array}$ \\
\hline Paraguay & 13.000 & 12.000 & $\begin{array}{r}1.000 \\
(7,7)\end{array}$ & $\begin{array}{l}8.200 \\
(63,1)\end{array}$ & $\begin{array}{l}3.800 \\
(29,2)\end{array}$ & 1.973 & $\begin{array}{r}646 \\
(32,7)\end{array}$ & $\begin{array}{l}1.327 \\
(67,3)\end{array}$ \\
\hline Perú & 75.000 & 73.000 & $\begin{array}{r}2.000 \\
(2,7)\end{array}$ & $\begin{array}{r}55.000 \\
(73,3)\end{array}$ & $\begin{array}{r}18.000 \\
(24,0)\end{array}$ & 8.481 & $\begin{array}{l}2.779 \\
(32,8)\end{array}$ & $\begin{array}{l}5.702 \\
(67,2)\end{array}$ \\
\hline $\begin{array}{l}\text { República } \\
\text { Dominicana }\end{array}$ & 57.000 & 54.000 & $\begin{array}{r}3.000 \\
(5,3)\end{array}$ & $\begin{array}{r}22.000 \\
(38,6)\end{array}$ & $\begin{array}{r}32.000 \\
(56,1)\end{array}$ & 9.391 & $\begin{array}{l}6.553 \\
(69,8)\end{array}$ & $\begin{array}{l}2.838 \\
(30,2)\end{array}$ \\
\hline Uruguay & 9.900 & 9.600 & $\begin{array}{r}300 \\
(3,0)\end{array}$ & $\begin{array}{l}6.500 \\
(65,6)\end{array}$ & $\begin{array}{l}3.100 \\
(31,4)\end{array}$ & 1.294 & $\begin{array}{r}507 \\
(39,2)\end{array}$ & $\begin{array}{r}787 \\
(60,8)\end{array}$ \\
\hline Venezuela & - & - & - & - & - & - & - & - \\
\hline
\end{tabular}


jóvenes con VIH/SIDA. En cuanto a los varones jóvenes, el grupo de países formado por Colombia $(68,2 \%)$, Costa Rica $(68,0 \%)$ y Paraguay $(67,3 \%)$ lidera el porcentaje de personas de este grupo que padecen el VIH/SIDA. Bolivia (8,3\%), Paraguay $(7,7 \%)$ y Colombia $(6,3 \%)$ constituyen el conjunto de países latinoamericanos con mayor porcentaje de casos infantiles afectados por el VIH/SIDA (véase Tabla 3 ).

Las vías de transmisión del VIH se pueden apreciar en la Tabla 4. La vía predominante en todos los países latinoamericanos es la sexual (heterosexual y homo/bisexual). México, Panamá y, en menor medida Perú, son los países donde la vía hetero y homosexual se encuentran más igualadas en porcentajes. Los países con mayores porcentajes de infección por el VIH a través de la vía heterosexual son Honduras $(85,3 \%)$, Nicaragua $(81,0 \%)$ y Ecuador $(80,1 \%)$. La vía homo/ bisexual presenta los mayores porcentajes en Cuba $(72,0 \%)$, Venezuela $(65,0 \%)$ y Costa Rica $(59,3 \%)$. Con respecto al consumo de drogas por vía parenteral, Uruguay (19,0\%), Brasil (14,3\%) y Argentina (5,7\%) encabezan las posiciones de países donde se concentran los porcentajes más elevados. A pesar del control de las transfusiones sanguíneas, el 4,0\% de los casos de VIH en Costa Rica se ha producido a través de esta vía, así como el 3,0\% de los casos de República Dominicana y el 1,6\% de los de México (Tabla 4). Analizando la vía madre-hijo, se observa que Honduras $(6,1 \%)$, El Salvador $(6,0 \%)$ y Guatemala $(5,1 \%)$

Tabla 4. Vías de transmisión del VIH en los países latinoamericanos y España. Actualizado a marzo de 2010. Fuente: ONUSIDA ${ }^{18}$ y Centro Nacional de Epidemiología ${ }^{14}$

\begin{tabular}{|c|c|c|c|c|c|c|c|}
\hline País & $\begin{array}{c}\text { Sexual } \\
(\%)\end{array}$ & $\begin{array}{c}\text { Hetero } \\
(\%)\end{array}$ & $\begin{array}{l}\text { Homo/ } \\
\text { bi }(\%)\end{array}$ & $\begin{array}{l}\text { UDVP } \\
(\%)\end{array}$ & $\begin{array}{c}\text { Transf/hemo } \\
(\%)\end{array}$ & $\begin{array}{l}\text { M-H } \\
\text { (\%) }\end{array}$ & Otro/Desc (\%) \\
\hline Argentina & 90,8 & 68,3 & 22,5 & 5,7 & 0,6 & 2,8 & 0,1 \\
\hline *Bolivia & 90,0 & 67,5 & 14,4 & - & 1,0 & 2,0 & 7,0 \\
\hline Brasil & 72,6 & 50,8 & 21,8 & 14,3 & 0,7 & 0,1 & 12,3 \\
\hline Chile & 88,3 & 36,5 & 51,8 & 0,9 & 0,4 & 1,1 & 9,3 \\
\hline Colombia & 82,3 & 60,4 & 21,9 & 0,1 & - & 1,0 & 16,6 \\
\hline Costa Rica & 84,2 & 24,9 & 59,3 & - & 4,0 & 1,3 & 10,5 \\
\hline Cuba & 99,0 & 27,0 & 72,0 & - & - & - & 1,0 \\
\hline Ecuador & 100,0 & 80,1 & 19,9 & - & - & - & - \\
\hline El Salvador & 86,0 & 79,0 & 7,0 & 1,0 & - & 6,0 & 7,0 \\
\hline Guatemala & 94,1 & - & - & - & - & 5,1 & 0,8 \\
\hline Honduras & 91,8 & 85,3 & 6,5 & 0,1 & 0,4 & 6,1 & 1,6 \\
\hline México & 63,6 & 34,2 & 29,4 & 0,8 & 1,6 & - & 34,0 \\
\hline Nicaragua & 100,0 & 81,0 & 19,0 & - & - & - & - \\
\hline Panamá & 69,0 & 36,3 & 32,7 & - & 1,5 & 3,2 & 26,3 \\
\hline Paraguay & 98,0 & 70,2 & 27,8 & 0,9 & - & 1,1 & - \\
\hline Perú & 97,0 & 41,0 & 56,0 & - & 1,0 & 2,0 & - \\
\hline República Dominicana & 82,7 & 75,7 & 7,0 & 5,0 & 3,0 & - & 9,3 \\
\hline Uruguay & 70,0 & 51,2 & 18,8 & 19,0 & - & 2,6 & 8,4 \\
\hline Venezuela & 100,0 & 35,0 & 65,0 & - & - & - & - \\
\hline España & 31,4 & 17,2 & 14,2 & 60,9 & 1,5 & 1,2 & 4,9 \\
\hline
\end{tabular}

Nota: Hetero: Heterosexual; Homo/Bi: Homosexual/bisexual; Transf/Hemo: Transfusión sanguínea/hemoderivados; M-H: MadreHijo; Otro/Desc: Otro/Desconocido. Las casillas con guión (-) significan que no hay datos. *Bolivia: El $90 \%$ contrajo el VIH a través de la vía sexual y de este porcentaje, no se tiene datos acerca de la vía sexual exacta de infección en el 8,1\% de los casos. 


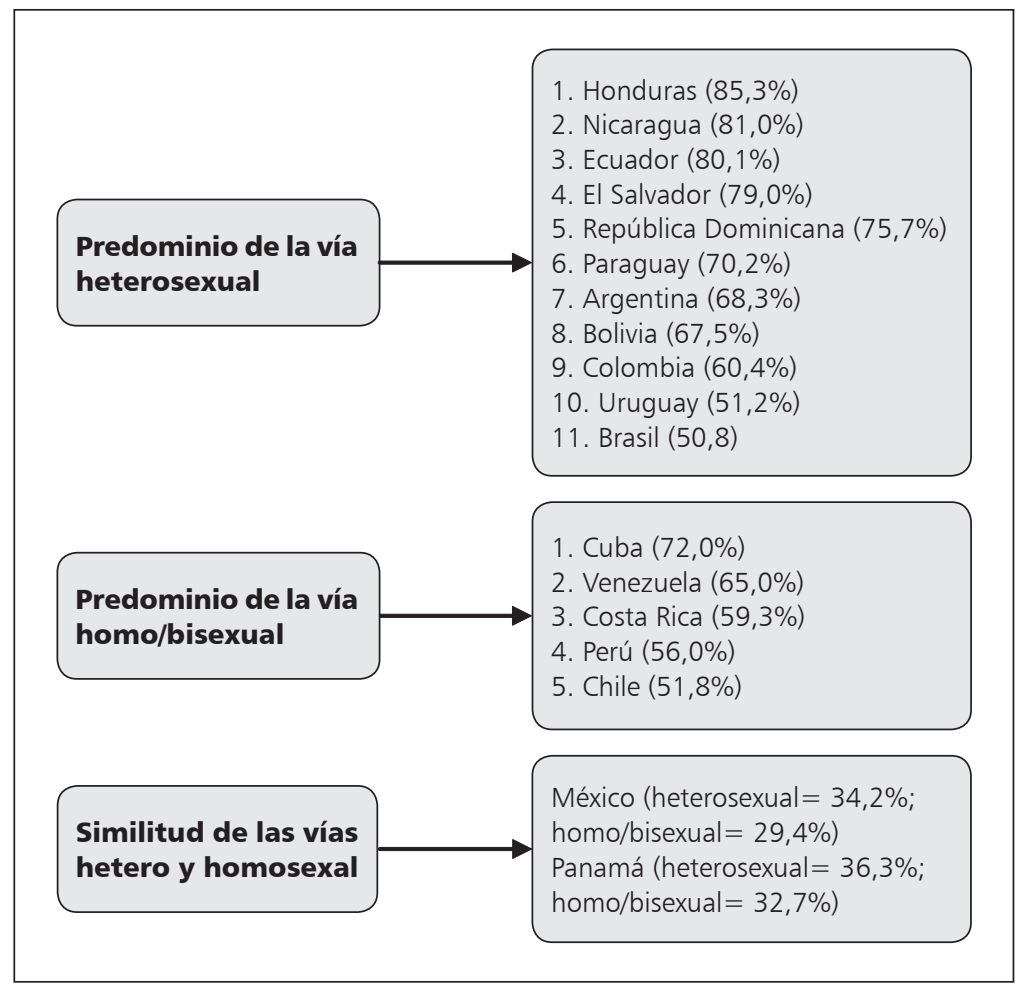

Figura 1. Clasificación de los países latinoamericanos en función de la vía de transmisión predominante. tienen la proporción más elevada de transmisión materno-filial en Latinoamérica. Existe un elevado porcentaje de casos sobre los cuales no se conoce la vía de transmisión. Así, México (34,0\%), Panamá $(26,3 \%)$ y Colombia $(16,6 \%)$ constituyen el grupo de tres países donde se concentran los porcentajes más elevados de casos de VIH cuya vía de transmisión se desconoce. En la Figura 1 se muestra una agrupación de los países en función de la vía de transmisión predominante. Como se indica, Guatemala no ha sido clasificado puesto que únicamente se conoce que $94,1 \%$ de los casos de VIH se debe a la vía sexual, no especificándose el desglose de porcentajes en función de la vía heterosexual y homo/bisexual.

\section{Discusión}

El Caribe y Latinoamérica son dos de las regiones con mayor tasa de prevalencia de $\mathrm{VIH} /$ SIDA en adultos. Se aducen como razones de las diferencias entre los índices de prevalencia de estas regiones factores religiosos, culturales, sociales, económicos y políticos (entre otros) que influyen sobre la conducta sexual así como en el nivel de desarrollo ${ }^{19,20}$.

A partir de los resultados obtenidos, se concluye que Latinoamérica es una región muy heterogénea con respecto a su tasa de prevalencia de VIH/SIDA. Esta falta de homogeneidad en las tasas de prevalencia ya ha sido puesta de manifiesto con anterioridad ${ }^{3}$. Teniendo en cuenta que la principal vía de transmisión en la República Dominicana es la heterosexual y que precisamente los países con prevalencia alta se caracterizan por el predominio de esta vía, se deduce que existe un riesgo de expansión del VIH a través de las relaciones heterosexuales, a pesar de que el mecanismo predominante de transmisión del VIH en la región sea la homosexual, según ONUSIDA² . Es más, República Dominicana, Nicaragua y Ecuador que son los países donde se acumulan los mayores porcentajes de mujeres jóvenes con VIH/SIDA, son también los lugares donde predomina la vía heterosexual. El turismo sexual de la región unido a las relaciones sexuales que los varones homosexuales mantienen con mujeres como forma de esconder su condición, podrían ser algunas explicaciones a 
la base de esta superioridad de la vía heterosexual. Otras razones podrían ser la violencia de género mediante el abuso sexual, el menor acceso a la información que tienen las mujeres y la necesidad de mejora de sus habilidades para la negociación del sexo protegido que exponen al riesgo de infección a las mujeres. Precisamente, existen estudios muy actuales en los que se ha abordado la influencia de las dinámicas de poder y los roles de género en la pareja sobre el riesgo de infección de $\mathrm{VIH}^{21-23}$. Por tanto, se precisa el desarrollo de investigaciones y la implantación de programas de prevención del VIH y enfermedades de transmisión sexual en estos países que tengan en cuenta estos factores y se focalicen en esta población. Por otro lado, se resalta que la elevada prevalencia de VIH/SIDA en varones que tienen relaciones sexuales con varones se relacionaría también con el desconocimiento del tamaño de este grupo lo cual explicaría la baja o nula existencia de políticas públicas preventivas dirigidas a este sector.

Se pone de manifiesto que los países difieren entre sí en los porcentajes de transmisión maternofilial. A nivel regional, se puede afirmar que la vía de transmisión madre-hijo es elevada siendo muy heterogéneas las formas empleadas en cada país para evaluar el alcance de dicha vía ${ }^{24}$. Por tanto, las políticas sanitarias en este asunto no son homogéneas entre los países, lo cual explicaría los porcentajes tan dispares en esta vía de transmisión. En Guatemala y Honduras, dos de los países con los mayores porcentajes de casos de VIH/ SIDA debidos a esta vía, menos de la mitad de las mujeres embarazadas con un diagnóstico de VIH se encuentran en tratamiento para la infección ${ }^{24}$. Esto sería una razón de los elevados porcentajes de casos de VIH/SIDA debidos a la vía materno-filial en estos países.

En relación al consumo de drogas por vía parenteral, Uruguay, Brasil y Argentina son los países latinoamericanos con los mayores porcentajes de personas que se infectaron por este mecanismo. La magnitud de la transmisión sexual del VIH en Latinoamérica es tan grande que se considera secundaria la vía de transmisión a través del consumo de drogas por vía intravenosa, lo cual ha tenido como consecuencia una escasez de actividades destinadas a la prevención del VIH en drogodependientes ${ }^{24}$. Es más, en estudios recientes ${ }^{25}$ se ha puesto de manifiesto que en Latinoamérica existen datos escasos sobre la vía de transmisión a través del consumo de drogas por vía intravenosa y no consta que haya servicios de prevención y tratamiento para estas personas. Únicamente en Brasil y México se han introducido programas para la reducción de daños en drogodependientes ${ }^{25}$. Por tanto, los países latinoamericanos en general y más concretamente, Argentina, Uruguay y Brasil no están realizando las acciones pertinentes (o bien éstas son insuficientes) en materia de prevención y erradicación de este mecanismo de transmisión.

Pese a que existe una gran cantidad de información disponible acerca de las características del VIH/SIDA en los países latinoamericanos gracias a la implementación de la vigilancia de segunda generación, sin embargo, se dan carencias en algunos datos y la calidad de la información disponible difiere entre los países. Además, se encuentran porcentajes relevantes de casos sobre los cuales no se conoce la vía de transmisión. Por tanto, es precisa la mejora de la vigilancia epidemiológica del VIH/SIDA en Latinoamérica. Limitaciones similares ya han sido manifestadas con anterioridad en estudios realizados en la región ${ }^{4,19}$. Si bien no es posible hacer un análisis comparativo con el estudio de Buela-Casal et $\mathrm{al}^{3}$ puesto que en el presente trabajo se han analizado los casos de VIH/ SIDA mientras que en el de Buela-Casal et $\mathrm{al}^{3}$ se analizaron únicamente los casos de SIDA, cabe mencionar que los resultados que se presentan en el estudio actual suponen un avance puesto que se está considerando tanto a las personas que están infectadas como a aquellas que han desarrollado la enfermedad. Ello implica que se pueda tener una mejor perspectiva de la epidemia. Por otra parte, significa que se están dedicando esfuerzos a la mejora de los sistemas de vigilancia epidemiológica. Sin embargo, sería de gran utilidad disponer de los casos de VIH separados de los casos de SIDA, lo cual redundaría en un conocimiento más profundo de la evolución de la epidemia.

Se resalta el creciente interés y número de estudios que se están dedicando a la investigación y aplicación de programas de prevención del VIH en Latinoamérica y en poblaciones latinoamericanas $^{26-31}$. Por otra parte, no hay que olvidar la inmigración, que facilita la propagación del virus en los países de acogida. Por ejemplo, Bermúdez et $\mathrm{al}^{6}$ concluyeron que $81,6 \%$ de los casos de SIDA en extranjeros en España tiene lugar en personas africanas y latinoamericanas que son los colectivos de inmigrantes más numerosos y cuyos países 
de origen son aquellos donde se concentran las mayores tasas de prevalencia de VIH/SIDA. De ahí también la importancia del desarrollo e implementación de programas de prevención en estos países para frenar el crecimiento de la epidemia a nivel regional y global.

A modo de conclusión general, se afirma que el análisis de los países latinoamericanos en función de la prevalencia de VIH/SIDA, la distribución de casos en función del sexo, la edad y las vías de transmisión hace de Latinoamérica una región muy heterogénea. Este aspecto es de gran relevancia de cara a la investigación para el desarrollo e implantación de estrategias preventivas que deben adecuarse a las características particulares de cada país. Destacar dos líneas esenciales de actuación: la mejora de la vigilancia epidemiológica y la prevención.

\section{Referencias}

1. Kilmarx PH. Global epidemiology of HIV. Curr Opin HIV AIDS 2009; 4: 240-6.

2. ONUSIDA. Report on the global AIDS epidemic, 2010. Disponible en: http://www.unaids.org/globalreport/ Global_report.htm. Consultado el 12 de febrero de 2011.

3. Buela-Casal G, Bermúdez MP, Sánchez AI, De los Santos-Roig M. Situación del VIH/SIDA en Latinoamérica al final del siglo XX. Análisis de las diferencias entre países. Rev Med Chile 200; 129: 944-54.

4. Alonso González M, Rivero Montesdeoca, Y. Situación de la epidemia en Latino América tras 30 años de su inicio. Rev Temp Act Saúde Colet 2010; 4: 131-43.

5. Bastos FI, Cáceres C, Galvao J, Veras MA, Castilho EA. AIDS in Latin America: Assesing the current status of the epidemic and the ongoing response. Int J Epidemiol 2008; 37: 729-37.

6. Bermúdez MP, Castro A, Buela-Casal G. SIDA en España e inmigración: Análisis epidemiológico. Univ Psicol 2009; 8: 37-47.

7. Bermúdez MP, Teva I. Situación actual del VIH/SIDA en México: análisis de las diferencias por entidad federativa. Psicol Sal 2004; 14: 101-11.

8. Bermúdez MP, Teva-Álvarez I. Situación actual del VIH/ SIDA en Europa: análisis de las diferencias entre países. Int J Clin Health Psychol 2003; 3: 89-106.

9. Bermúdez MP, Teva I, Buela-Casal G. Situación actual del SIDA en España: análisis de las diferencias entre comunidades autónomas. Int J Clin Health Psychol 2004; 4: 553-70.
10. Hamers FF, Infuso A, Aux J, Downs AM. Current situation and regional perspective on HIV/AIDS surveillance in Europe. J Acquir Immune Defic Syndr 2003; 32: 39-48.

11. Hamers FF, Phillips AN. Diagnosed and undiagnosed HIV-infected populations in Europe. HIV Med 2008; 9: 6-12.

12. Pisani E, Lazari S, Walker N, Schwartländer B. HIV surveillance: A global perspective. J Acquir Immune Defic Syndr 2003; 32: 3-11.

13. Van de Laar MJ, Likatavicius G, Stengard AR, Donoghoe MC. HIV/AIDS Surveillance in Europe: Update 2007. Eurosurveillance 2008; 13: 1-3.

14. Centro Nacional de Epidemiología. Vigilancia Epidemiológica del SIDA en España. Actualización a 30 de junio de 2010. Disponible en: http://www.isciii.es/htdocs/ centros/epidemiologia/pdf/SPNS_Informe_semestral. pdf. Consultado el 21 de marzo de 2011.

15. Montero I, León OG. A guide for naming research studies in Psychology. Int J Clin Health Psychol 2007; 7: 847-62.

16. ONUSIDA. Epidemiological factsheet on HIV and AIDS, 2009. Disponible en: http://www.unaids.org/en/ regionscountries/countries. Consultado el 12 de febrero de 2011.

17. CELADE. Datos de población actualizados a septiembre de 2009. Disponible en: http://www.eclac.org/celade/ proyecciones/basedatos_BD.htm. Consultado el 12 de febrero de 2011.

18. ONUSIDA. Informes de progreso presentados por los países en 2010. Disponible en: http://www.unaids.org. Consultado el 3 de marzo de 2011.

19. García Calleja JM, Walker N, Cuchi P, Lazzari S, Ghys PD, Zacarías F. Status of the HIV/AIDS epidemic and method to monitor it in the Latin America and Caribbean region. AIDS 2002; 16: 3-12.

20. Zanakis SH, Álvarez C, Li V. Socio-economic determinants of VIH/AIDS pandemic and nations efficencies. Eur J Oper Res 2007; 176: 1811-38.

21. Bermúdez MP, Castro A, Gude F, Buela-Casal G. Relationship power in the couple and sexual double Standard as predictors of the risk of sexually transmitted infections and HIV: Multicultural and gender differences. Curr HIV Res 2010; 8: 172-8.

22. Manji A, Peña R, Dubrow, R. Sex, condoms, gender roles and HIV transmisión knowledge among adolescents in León, Nicaragua: Implications for HIV prevention. AIDS Care 2007; 19: 989-95.

23. Pulerwitz J, Michaelis A, Verma R, Weiss E. Addressing gender dynamics and engaging men in HIV programs: Lessons learned from horizons research. Public Health Rep 2010; 125: 282-92. 
24. ONUSIDA Análisis regional consolidado de los informes UNGASS presentados por 17 países de América Latina en 2010. Disponible en: http://www.onusida-latina.org/ index.php?item $=2 \&$ content $=$ article\&id $=18$. Consultado el 3 de octubre de 2011.

25. Mathers BM, Degenhardt L, Ali H, Wiessing L, Hickman M, Mattick RP, et al. HIV prevention, treatment, and care services for people who inject drugs: A systematic review of global, regional, and national coverage. Lancet 2010; 375: 1014-28.

26. Albarracín J, Albarracín D, Durantini M. Effects of HIV prevention interventions for simples with higher and lower percents of Latinos and Latin Americans: A metaanalysis of change in condom use and knowledge. AIDS Behav 2008; 12: 521-43.

27. Demaria LM, Galárraga O, Campero L, Walter DM. Educación sobre sexualidad y prevención del VIH: Un diagnóstico para América Latina y el Caribe. Rev Panam
Salud Publica 2009; 26: 485-93.

28. Fernández MI, Nelly JA, Stevenson LY, Sitzler CA, Hurtado J, Vladez C, et al. HIV prevention programs of nongovernmental organizations in Latin America and the Caribbean: The Global AIDS Intervention Network project. Rev Panam Salud Publica 2005; 17: 154-62.

29. Huedo-Medina TB, Boynton MH, Warren MR, Lacroix JM, Carey MP, Johnston BT. Efficacy of HIV prevention interventions in Lation American and Caribbean nations, 1995-2008: A meta-analysis. AIDS Behav 2010; 14: 1237-51.

30. Johri M, Morales RE, Hoch JS, Samayoa BE, Sommen C, Grazioso CF, et al. A cross-sectional study of risk factors for HIV among pregnant women in Guatemala City, Guatemala: Lessons for prevention. Int J STD AIDS 2010; 21: 789-96.

31. Pérez F, Dabis F.HIV prevention in Latin America: Reaching youth in Colombia. AIDS Care 2003; 15: 77-87. 